\title{
Structure of the Mediator Subunit Cyclin C and its Implications for CDK8 Function
}

\section{Sabine Hoeppner, Sonja Baumli and Patrick Cramer*}

Gene Center, University of Munich (LMU), Department of Chemistry and Biochemistry Feodor-Lynen-Str. 25, 81377 Munich, Germany
Cyclin $\mathrm{C}$ binds the cyclin-dependent kinases CDK8 and CDK3, which regulate mRNA transcription and the cell cycle, respectively. The crystal structure of cyclin $C$ reveals two canonical five-helix repeats and a specific $\mathrm{N}$-terminal helix. In contrast to other cyclins, the N-terminal helix is short, mobile, and in an exposed position that allows for interactions with proteins other than the CDKs. A model of the CDK8/cyclin C pair reveals two regions in the interface with apparently distinct roles. A conserved region explains promiscuous binding of cyclin $C$ to $C D K 8$ and $C D K 3$, and a non-conserved region may be responsible for discrimination of CDK8 against other CDKs involved in transcription. A conserved and cyclin C-specific surface groove may recruit substrates near the CDK8 active site. Activation of CDKs generally involves phosphorylation of a loop at a threonine residue. In CDK8, this loop is longer and the threonine is absent, suggesting an alternative mechanism of activation that we discuss based on a CDK8-cyclin C model.

(C) 2005 Elsevier Ltd. All rights reserved.

Keywords: RNA polymerase II transcription; carboxy-terminal repeat domain; coactivator; cell cycle; cyclin-dependent kinase
The CDK8/cyclin $\mathrm{C}$ pair has a role in transcriptional repression. ${ }^{14}$ CDK8 was suggested to phosphorylate the CTD prematurely, thereby preventing formation of a transcription initiation complex. ${ }^{14}$ In human cells, CDK8/cyclin C also phosphorylates cyclin $\mathrm{H}$, repressing CDK7 activity and transcription. ${ }^{15}$ Further, CDK8 phosphorylates certain gene-specific transcription factors and decreases their stability. ${ }^{16,17}$ However, there are some reports of a positive effect of CDK8/cylin C on transcription. CDK8-dependent phosphorylation of the gene-specific transcription factor Sip4 can stimulate transcription. ${ }^{18}$ CDK8 binds the Gal4 activation domain. ${ }^{19}$ CDK8 promotes ATP-dependent dissociation of preinitiation complexes, resulting in a positive effect on transcription. ${ }^{20} \mathrm{CDK} 8 /$ cyclin $C$ phosphorylates subunits of the general transcription factor TFIID, ${ }^{20}$ and the Mediator subunit MED2. ${ }^{21}$ Mutation of the MED2 phosphorylation site reduces expression levels of certain genes. $^{21}$

In addition to its role in transcription, cyclin $C$ is involved in cell cycle control. Cyclin C was identified originally in genetic screens for proteins that rescue cell cycle defects in yeast. ${ }^{22,23}$ The role of cyclin $C$ in cell cycle regulation has been elucidated recently. It was discovered that cyclin $C$ forms a complex with $\mathrm{CDK} 3$, and that the $\mathrm{CDK} 3$ / cyclin $\mathrm{C}$ 
pair is a key regulator of cell cycle re-entry in human cells. ${ }^{24}$

Structural information on $\mathrm{CDK} /$ cyclin pairs involved in transcription is limited to crystal structures of human CDK7 and cyclin $\mathrm{H}^{26,27}$ We report here the crystal structure of cyclin $\mathrm{C}$ from Schizosaccharomyces pombe. The structure reveals canonical cyclin repeat folds and specific features that may be relevant for assembly of the CDK8/ cyclin $C$ module, for kinase substrate recognition, and for CDK8 activation.

\section{Results and Discussion}

\section{Structure determination}

Purified recombinant full-length cyclin C from S. pombe formed small fragile crystals that diffracted synchrotron radiation to $3.5 \AA$ resolution. To improve crystal quality, we subjected the protein to limited proteolysis. Treatment with subtilisin resulted in cleavage of four or 29 residues from the cyclin $\mathrm{N}$ terminus. A protein variant that lacked the N-terminal 29 residues was insoluble, but a variant truncated by four residues produced crystals of improved stability that diffracted to $3.0 \AA$ resolution. Molecular replacement with known cyclin structures failed. The structure was therefore solved by multiwavelength anomalous diffraction (MAD) analysis with a selenomethionine-substituted crystal of a mutant protein that carried replacements of two leucine residues by methionine (Table 1). Using native diffraction data to $3.0 \AA$ resolution, the structure was refined to a free $R$-factor of $28.8 \%$ (Table 1 ). The final model shows excellent stereochemistry and comprises all residues of cyclin $C$, except residues 1-5 (Table 1).

\section{Canonical cyclin repeats}

The structure of cyclin $\mathrm{C}$ reveals two typical cyclin repeats consisting of five helices each $(\mathrm{H} 1-\mathrm{H} 5$ and $\mathrm{H} 1^{\prime}-\mathrm{H} 5^{\prime}$ in repeats 1 and 2 , respectively) (Figure 1). The length of some helices differs from that in cyclins $\mathrm{A}$ and $\mathrm{H}$ by several residues (Figure 2). The individual cyclin repeats 1 and 2 can be superimposed on those of cyclin $\mathrm{H}$ with a root-mean-square deviation (RMSD) in main-chain atom positions of $1.6 \AA$ and $2.5 \AA$, respectively. The relative orientation of the two cyclin repeats is also similar to that observed in cyclins $\mathrm{A}$ and $\mathrm{H}$, so that both repeats of cyclin $\mathrm{C}$ can be superimposed with an RMSD of $2.7 \AA$ in each case. The similar orientation of the two repeats in cyclins $\mathrm{C}, \mathrm{H}$ and A apparently results from conservation of the repeat interface, which involves residues from helices $\mathrm{H} 1, \mathrm{H} 2, \mathrm{H}^{\prime}, \mathrm{H}_{2}^{\prime}$, and the conserved interrepeat linker (Figure 2). In particular, the highly conserved residue $\mathrm{R} 62$ in $\mathrm{H} 2$ binds to the linker backbone carbonyl group of residue F132 in the inter-repeat linker, as observed in cyclin $\mathrm{H}^{26}$ and to the carbonyl group of I134. In addition, residue Q50 in helix H1, Y139, and N164 in H2' form a cluster at the repeat interface. In contrast, the two cyclin repeats in the general transcription factor TFIIB adopt a different relative orientation. ${ }^{28}$

Table 1. Structure determination and refinement

\begin{tabular}{|c|c|c|c|c|}
\hline & $\begin{array}{c}\text { Cyclin C (5-228) variant } \\
\text { L60M/L186M, SeMet } \\
\text { MAD }\end{array}$ & & & Wild type, native \\
\hline \multicolumn{5}{|l|}{ A. Data collection } \\
\hline Space group & $P 3_{1} 21$ & & & $P 3_{1} 21$ \\
\hline \multicolumn{5}{|l|}{ Unit cell axes } \\
\hline$a(\AA)$ & 91.9 & & & 91.7 \\
\hline$b(\AA)$ & 91.9 & & & 91.7 \\
\hline$c(\AA)$ & 90.9 & & & 90.1 \\
\hline Wavelength $(\AA)$ & 0.97977 (peak) & 0.98004 (inflection) & 0.94927 (remote) & 1.0725 \\
\hline Resolution $(\AA)$ & $20-3.5(3.6-3.5)^{\mathrm{a}}$ & $20-3.5(3.6-3.5)$ & $20-3.5(3.6-3.5)$ & $20-3.0(3.16-3.0)$ \\
\hline Completeness (\%) & $99.4(100)$ & $99.5(100)$ & $99.7(100)$ & $99.4(99.4)$ \\
\hline Unique reflections & $5866(581)$ & $5873(581)$ & $5885(581)$ & 9161 (1333) \\
\hline Redundancy & 9.12 & 9.04 & 9.29 & 6.0 \\
\hline$R_{\text {sym }}(\%)$ & $8.6(28.8)$ & $7.4(35.9)$ & $5.8(27.7)$ & $8.1(41.6)$ \\
\hline Mean $I / \sigma I$ & $23.6(7.3)$ & $23.8(6.6)$ & $24.5(6.1)$ & $17.0(3.0)$ \\
\hline$f^{\prime}$ & -7.0 & -10.2 & -2.8 & - \\
\hline$f^{\prime \prime}$ & 5.5 & 3 & 3.6 & - \\
\hline \multicolumn{5}{|l|}{ B. Refinement } \\
\hline Residues & & & & $227^{\mathrm{b}}$ \\
\hline \multicolumn{5}{|l|}{ RMSD from ideal } \\
\hline Bond lengths (§) & & & & 0.008 \\
\hline Bond angles (deg.) & & & & 1.3 \\
\hline$R_{\text {cryst }}(\%)$ & & & & 24.4 \\
\hline$R_{\text {free }}(\%)$ & & & & 28.8 \\
\hline
\end{tabular}


(a)

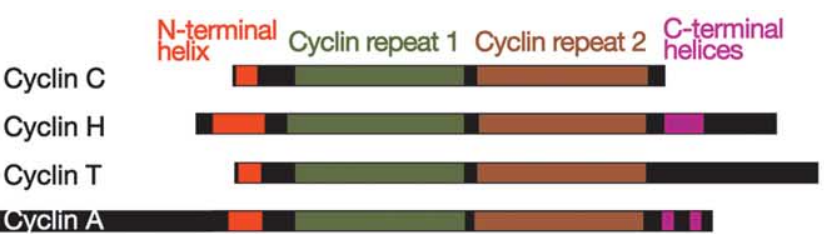

(c)

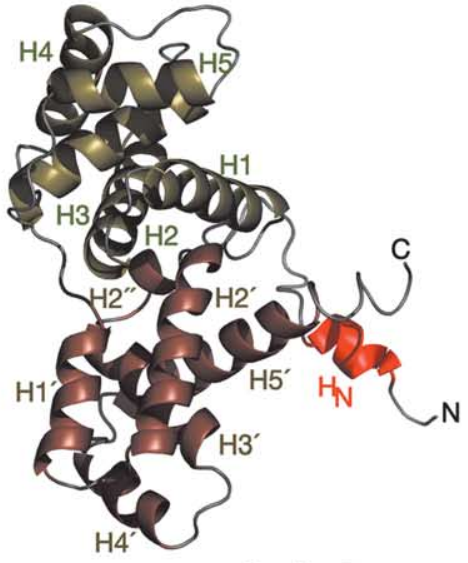

Cyclin C

(d)

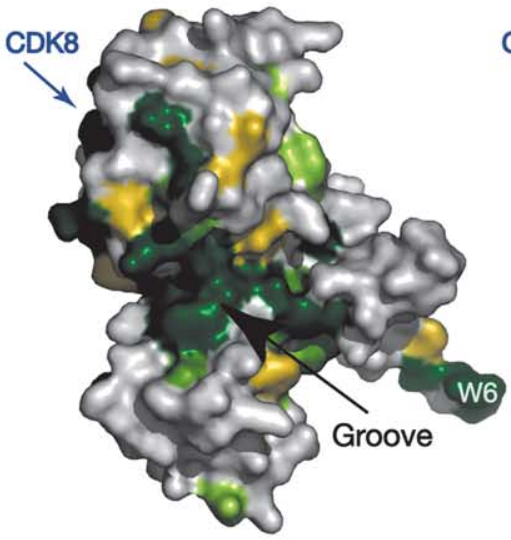

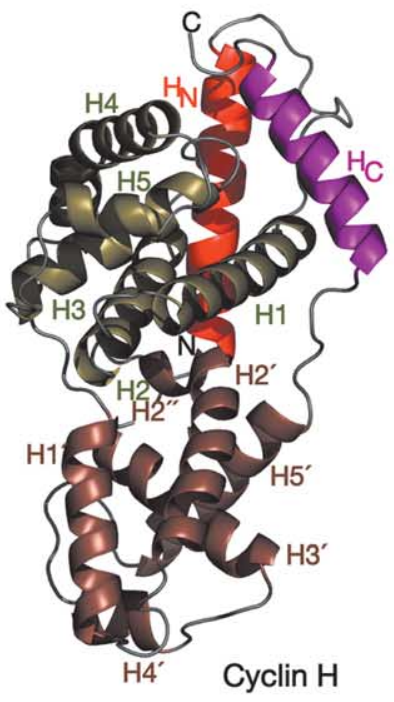

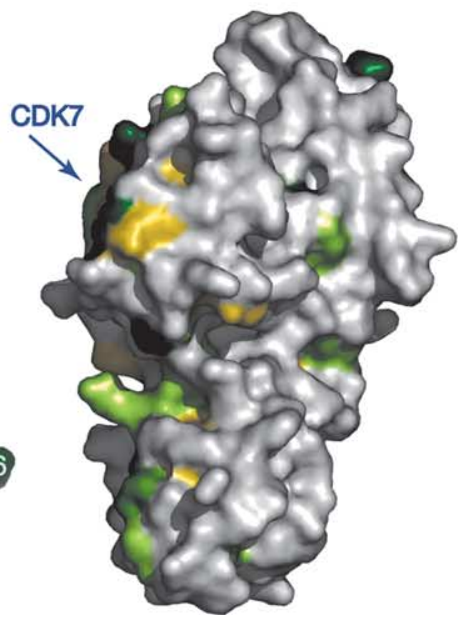

(b)
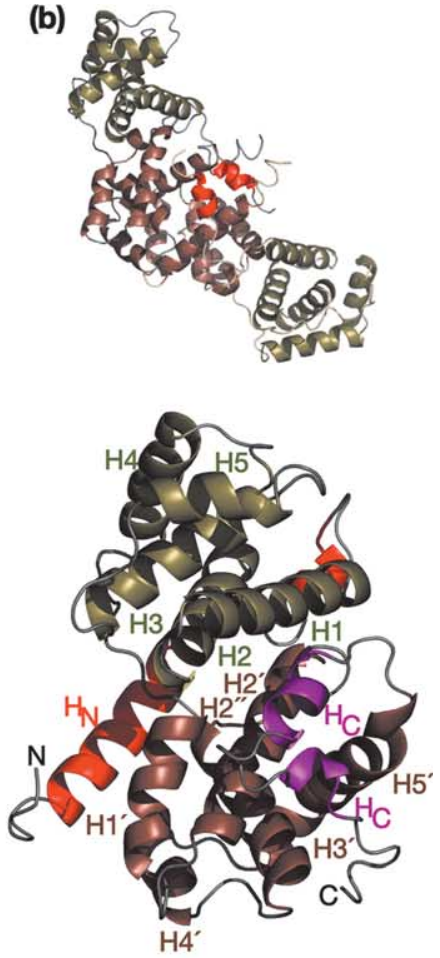

Cyclin A

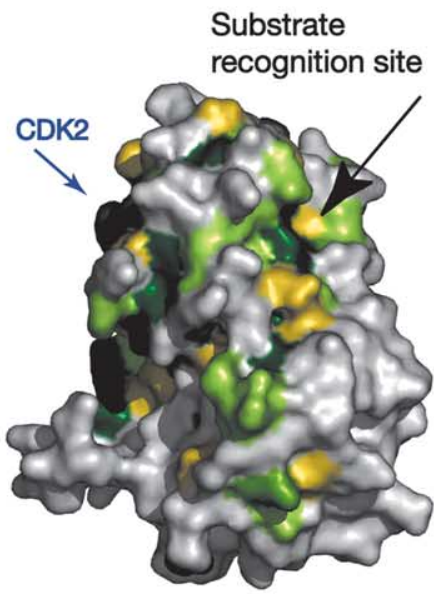

Figure 1. Structure of cylin C and comparison with cyclins A and H. (a) Schematic diagram of cyclin primary structures. The two canonical cyclin repeats and the $\mathrm{N}$ and $\mathrm{C}$-terminal helices are highlighted. Bars are drawn to scale. (b) Ribbon model of the crystallographic dimer of S. pombe cyclin C. (c) Structural comparison of cyclins C, $\mathrm{H}^{26}$ (PDBcode $1 \mathrm{JKW}$ ), and $\mathrm{A}^{30}$ (PDB-code $\left.1 \mathrm{FIN}\right)$. The proteins are drawn as ribbon models. The colour code for secondary structure elements is as in A and in Figure 2. Helices are numbered according to Figure 2. (d) Surface conservation. The surface of the three cyclins in $\mathrm{C}$ is coloured according to conservation as indicated in Figure 2. Residues are highlighted in dark green, green, light green, and yellow, according to decreasing degree of conservation. The view is as in (c). Figures prepared with PYMOL (DeLano Scientific).

\section{A mobile N-terminal helix}

Cyclin C differs from known cyclin structures mainly outside the canonical repeats. Before the first repeat, cyclin $\mathrm{C}$ contains only one $\mathrm{N}$-terminal helix $\left(\mathrm{H}_{\mathrm{N}}\right)$, whereas cyclin $\mathrm{A}$ contains also an extended N-terminal region (Figure 1). The $\mathrm{C}$ terminus of cyclin $\mathrm{C}$ is formed by the last helix in the second repeat, whereas cyclins $\mathrm{H}$ and $\mathrm{A}$ comprise additional C-terminal helices. Cyclin C may thus be regarded as a minimal cyclin consisting only of the two canonical repeats and a specific $\mathrm{H}_{\mathrm{N}}$ helix.

Helix $\mathrm{H}_{\mathrm{N}}$ in cyclin $\mathrm{C}$ differs from that in cyclins $\mathrm{A}$ and $\mathrm{H}$ in three aspects (Figures 1(c) and 2). The helix is much shorter, adopts a different position, and is mobile. The $\mathrm{H}_{\mathrm{N}}$ helix protrudes from the cyclin repeats, whereas the N-terminal helices of 

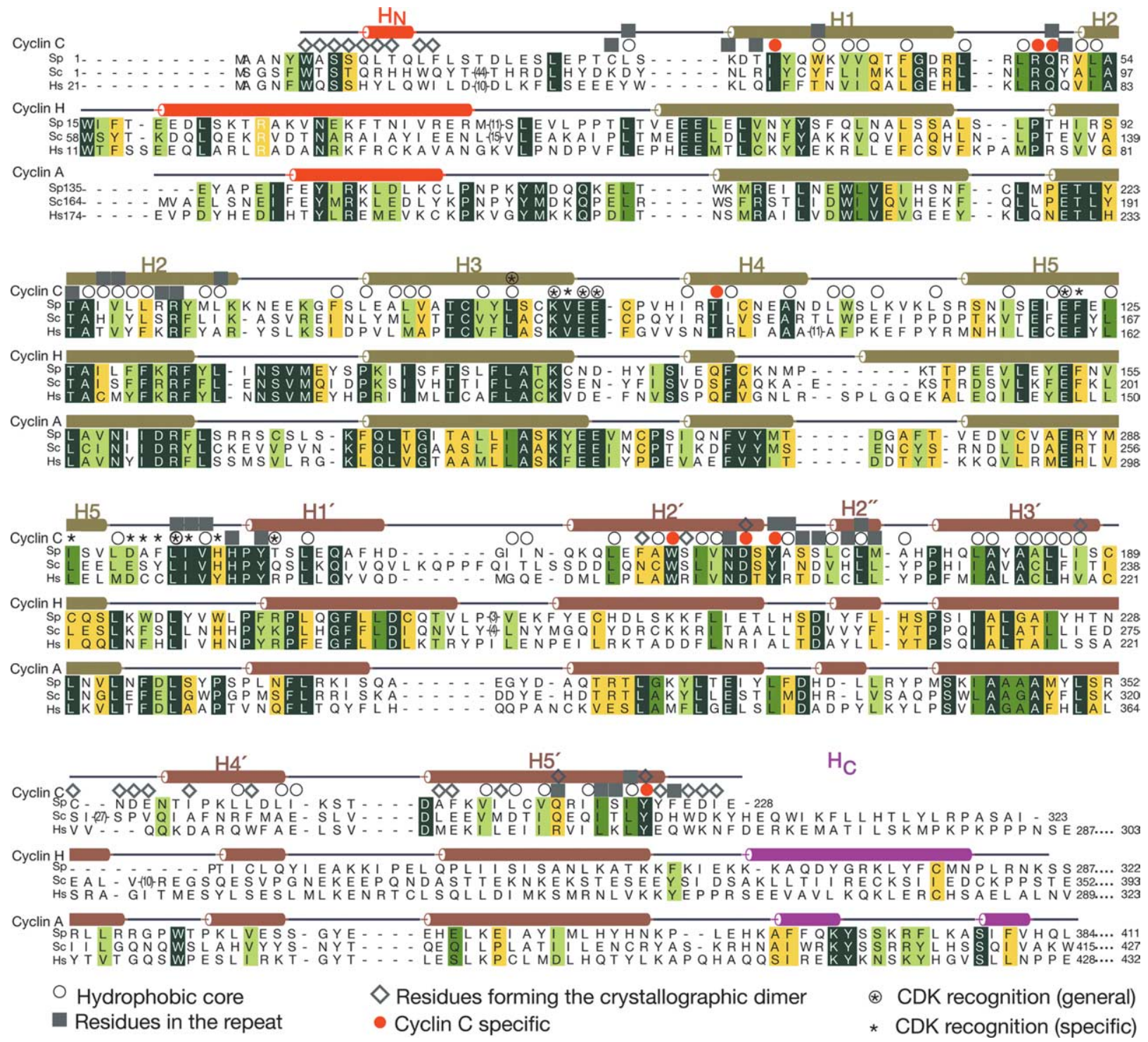

Figure 2. Structure-based sequence alignment of cyclin classes $\mathrm{C}, \mathrm{H}$, and A. Structure-based alignment of protein sequences of $S$. pombe cyclin $\mathrm{C}$ (this study) with human cyclin $\mathrm{H}_{r}{ }^{26}$ and human cyclin $\mathrm{A} \cdot{ }^{30}$ Alignments were extended with CLUSTAL W ${ }^{46}$ to three members of each cyclin family, from S. pombe (Sp), S. cerevisiae (Sc), and Homo sapiens (Hs), and were edited by hand. Helical regions were determined with $\operatorname{DSSP}^{47}$ and are shown above the alignments as cylinders. Residues are highlighted in dark green, green, light green, and yellow, according to decreasing degree of conservation. Residues in the structural core, the repeat interface, and the crystallographic dimer contact are marked with open circles, filled squares, and open diamonds, respectively. Residues that are strictly conserved and at the same time specific for cyclin $C$ are marked with a red dot. Residues involved in CDK recognition are marked with asterisks (compare Table 2). Prepared with ALSCRIPT. ${ }^{48}$

cyclins $\mathrm{H}$ and $\mathrm{A}$ pack intimately onto the repeats with conserved residues (Figure 1 (c) and (d)). ${ }^{26}$ The cyclin $\mathrm{C}$ helix $\mathrm{H}_{\mathrm{N}}$ and the subsequent loop show poor electron density, and have $B$-factors well above the average $B$-factor for the two cyclin repeats (on average, $33 \AA^{2}$ for residues 6-29; $75 \AA^{2}$ for residues $30-228$ ). However the $\mathrm{N}$-terminal helices in cyclins $\mathrm{H}$ and A show $B$-factors that are comparable to the overall $B$-factors of the structures. Also, partial proteolysis of cyclin $\mathrm{H}$ does not cleave its N-terminal helix, indicating that it is an integral part of the structure. The rigid nature of the $\mathrm{N}$-terminal helices in cyclin A, and in a viral cyclin, is indicated further by the observation that they adopt the same position in the free cyclins and in their complexes with CDK2. ${ }^{29,30}$

\section{Structure-based alignments}

Using the structures of cyclin C (this work), cyclin $\mathrm{H}^{26}$ and cyclin $\mathrm{A},{ }^{30}$ we prepared a structure-based alignment of these different cyclin families using sequences from S. pombe, Saccharomyces cerevisiae, and human (Figure 2). The structure-based alignment allowed us to identify residues that occur only in a certain type of cyclin, and thus are candidates 
for cyclin-specific functions (see below). The sequence comparisons revealed that cyclin $C$ is the most conserved of all cyclins. Human cyclin $\mathrm{C}$ shares $71 \%$ and $29 \%$ of its residues with cyclin C from Drosophila melanogaster and S. cerevisiae, respectively, whereas the corresponding values for identical residues are $32 \%$ and $21 \%$ in cyclin $\mathrm{A}$, and $40 \%$ and $26 \%$ in cyclin $\mathrm{H}$, respectively. In particular, the conservation of the second cyclin repeat is much more extended in cyclin $\mathrm{C}$ compared to that of other cyclins.

\section{Modelling of the CDK8-cylin C pair}

We obtained a model of the CDK8-cyclin C complex in two steps (Figure 3). First, we replaced cyclin A in the human CDK2-cyclin A complex crystal structure (PDB-code 1FIN) with our S. pombe cyclin $C$ structure. ${ }^{30}$ To this end, we superimposed residues in cyclin $C$ that are near the CDK interface (residues 85-89, 94-98, and 118-129) onto their counterparts in cyclin A (residues 263-267, 273-277, and 291-302, respectively). Second, we constructed a homology model of CDK8 by replacing all differing CDK2 residues with program O. ${ }^{31}$ Homology modelling was enabled by the high degree of sequence identity between $S$. pombe CDK8 and human CDK2 (37\%).

\section{Specificity of CDK-cyclin interaction}

Comparison of the CDK-cyclin interfaces in the CDK8-cyclin C model and the CDK2-cyclin A structure identified several highly conserved residues that are generally involved in CDK-cyclin interactions ("general," Table 2, Figure 3). Five CDK residues are involved in these generally conserved interactions with cyclin residues (V54, I59, R60, R160, L170 in S. pombe CDK8, Table 2), consistent with previous observations. ${ }^{26,30}$ The general

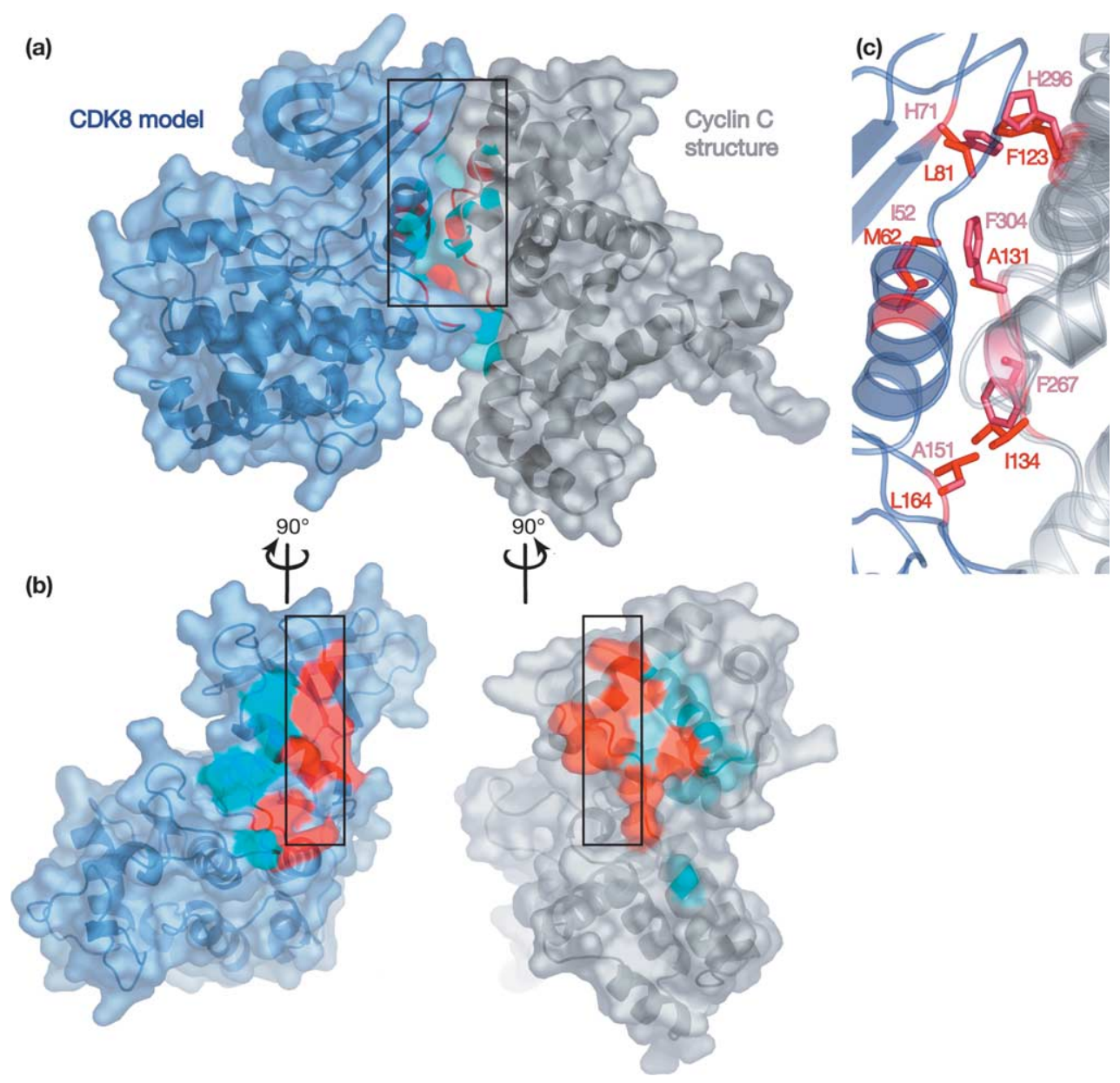

Figure 3. CDK-cyclin recognition. (a) Model of the CDK8-cyclin C complex, based on the CDK2-cyclin A structure (PDB-code 1FIN). ${ }^{30}$ The proteins are represented as ribbon models with a superimposed semitransparent molecular surface. The black box outlines the region of the complex depicted in (c). (b) "Book view" of the model in (a). The two protein representations were rotated by $90^{\circ}$ in opposite directions to allow viewing of the interface. Surfaces of residues that constitute the "general" and the "specific recognition region" of the CDK-cyclin interface were highlighted in turquoise and red, respectively (see the text and Table 2). (c) Comparison of selected residues in the specific recognition region of two CDK-cyclin interfaces. Residues of the CDK2-cyclin A structure (PDB-code 1FIN) are in light pink, and residues in the CDK8-cyclin $\mathrm{C}$ model are in red. 
Table 2. CDK-cyclin interactions

\begin{tabular}{|c|c|c|c|}
\hline CDK2 structure ${ }^{26}$ & CDK8 model & Cyclin A structure ${ }^{26}$ & Cyclin C structure \\
\hline \multicolumn{4}{|l|}{ A. General recognition region } \\
\hline \multirow[t]{2}{*}{ V44 (backbone) } & V54 (backbone) & K266 & K88 \\
\hline & & E295 & E122 \\
\hline \multirow[t]{3}{*}{ I49 } & I59 & K266 & K88 \\
\hline & & L263 & L85 \\
\hline & & L306 & L133 \\
\hline \multirow[t]{2}{*}{ R50 } & R60 & F267 (backbone) & V89 (backbone) \\
\hline & & K266 (backbone) & K88 (backbone) \\
\hline \multirow[t]{3}{*}{ R150 } & R164 & F267 (backbone) & V89 (backbone) \\
\hline & & E268 (backbone) & E90 (backbone) \\
\hline & & E269 (backbone) & E91 (side-chain) \\
\hline \multirow[t]{2}{*}{ V154 } & L170 & T316 & T140 \\
\hline & & E268 & E90 \\
\hline \multicolumn{4}{|l|}{ B. Specific recognition region } \\
\hline \multirow[t]{2}{*}{$\mathrm{H} 71$} & L81 & H296 & F123 \\
\hline & & - & I126 \\
\hline I52 & M62 & F304 & A131 \\
\hline \multirow{4}{*}{ S53 } & M63 & F304 (backbone) & A131 \\
\hline & & F267 & V89 \\
\hline & & L306 & L133 \\
\hline & & - & F132 (backbone) \\
\hline \multirow[t]{3}{*}{ K56 } & R66 & Т303 & $\mathrm{D} 130^{\mathrm{a}}$ \\
\hline & & F304 & A131 \\
\hline & & D305 ${ }^{2}$ & - \\
\hline \multirow[t]{2}{*}{ A151 } & L164 & A307 & I134 \\
\hline & & F267 & - \\
\hline \multirow[t]{2}{*}{ G153 } & R166 & Q313 & $\mathrm{T} 140$ \\
\hline & & - & H136 \\
\hline
\end{tabular}

Listed are CDK residues that have at least one atom within $4 \AA$ of an atom of the cognate cyclin. For the general recognition region, cyclin residues are included only if the contacts are observed in the CDK2-cyclin A structure and in the CDK8-cyclin C model.

a Possible alternative salt-bridges, see the text.

interactions explain why cyclin $C$ can interact with both CDK8 and CDK3. ${ }^{24}$ In addition, we identified residues in the $C D K-$ cyclin interface that are conserved only within a certain CDK or cyclin family. Theses "specific" residues may govern preferential interaction of a certain cyclin with a certain CDK (Table 2). The two classes of interface residues cluster in two regions, a "general" and a "specific recognition region" (turquoise and red, respectively, in Figure 3(b)).

Specficity of cyclin C for CDK8, as opposed to CDK2 or the two other CDKs involved in transcription, CDK7 and CDK9, may be explained by several contacts of side-chains in the specific recognition region. In particular, several hydrophobic sidechains that pack against each other in the interface of the complex model are complementary in size (Figure 3(c)). For example, the side-chains of L81 in the CDK8 model and F123 in the cyclin C structure contact each other, and correspond to the contact H71-H296 in the CDK2-cyclin A complex structure (Figure 3(c); Table 2). Residues M62 in the CDK8 model and A131 in the cyclin C structure also contact each other, and the corresponding contact I52-F304 is observed in the CDK2-cyclin A interface (Figure 3(c); Table 2). Similar complementary and specific pairs of residues are observed at these positions in sequences of the pairs CDK7-cyclin $\mathrm{H}$ and CDK9-cyclin T (Figure 2).

This analysis does not account for possible alternative interactions and rearrangements in the CDK-cyclin interface, which may be crucial to optimize the surface complementarity. For example, cyclins $\mathrm{A}$ and $\mathrm{C}$ apparently form alternative saltbridges at the interface with their cognate CDKs. A conserved basic residue in the kinase (K56 in CDK2, R66 in CDK8) is bound by the nonconserved aspartate D305 in cyclin A, but may be bound by D130 in cyclin C, which is offset in register by two residues (Table 2 ). Indeed side-chain rearrangements in a CDK-cyclin interface have been observed in the recent structure of a CDK2cyclin E1 complex, ${ }^{32}$ in which K56 of CDK2 adopts a different conformation and does not interact with the basic residue R225 in cyclin E1.

\section{Conserved surfaces}

Specificity for CDK-cyclin interaction may arise from additional protein subunits that could bridge between the kinase and the cyclin, such as MED12 (Srb8) and MED13 (Srb9) in the case of the CDK8cyclin $\mathrm{C}$ pair. To detect possible interaction sites for other proteins on cyclins $\mathrm{C}, \mathrm{H}$, and $\mathrm{A}$, their molecular surfaces were coloured according to conservation over species (Figure 1(d)). A conserved and cyclin $\mathrm{C}$-specific surface patch is found just before the mobile helix $\mathrm{H}_{\mathrm{N}}$, comprising the exposed aromatic residues Y5 and W6 (Figure 1(d)). Whereas the N-terminal helix of cyclin A contributes to the interface with $\mathrm{CDK} 2$, helix $\mathrm{H}_{\mathrm{N}}$ in cyclin $C$ points away from the kinase-binding surface (Figures 1 and 3 ), and cannot contribute to CDK8 binding. In the crystals, the N-terminal 
region of one molecule contacts the second cyclin repeat of its neighbour, resulting in a crystallographic dimer that buries several hydrophobic surface residues in its interface (L186, L200, A208, F209, I212, V215 and I219) (Figures 1(b) and 2). The dimer may pre-exist in solution, since deletion of helix $\mathrm{H}_{\mathrm{N}}$ results in an insoluble protein, likely because hydrophobic residues in the dimer interface are exposed (not shown). Since most of the hydrophobic residues and the $\mathrm{H}_{\mathrm{N}}$ helix are not conserved, the dimer is apparently not physiological. In the intact $\mathrm{CDK} 8 /$ cyclin $\mathrm{C}$ module, the $\mathrm{N}$-terminal region of cyclin $\mathrm{C}$ could therefore bind MED12 (Srb8) or MED13 (Srb9) and, given its mobility, may change its position upon interaction with the target protein.

\section{A conserved groove unique to cyclin C}

Cyclin C shows a highly conserved surface groove between the two cyclin repeats (Figure 1(d)). The corresponding region in cyclins $\mathrm{A}$ and $\mathrm{H}$ is not conserved, and the conserved groove in cyclin $\mathrm{C}$ is thus likely to have a function specific for this type of cyclin. Indeed, five of the surface residues in this groove are invariant among cyclin $\mathrm{C}$ family members, but are not conserved and do not occur in any other cyclin family (residues I33, R49, W160, D165 and Y167) (Figure 2, red dots, and Figure 4(a)). Since the groove is near the active site and the activation segment of CDK8 in the CDK8-cyclin C model (Figure 4), it may bind substrates and could contribute to CDK8 specificity. Indeed, cyclin A has a conserved surface patch that binds kinase substrates, albeit at a different location (Figure 1(d)). ${ }^{33}$ Recent work demonstrated the importance of this patch for substrate specificity. ${ }^{34}$ In contrast to the substrate recognition patch in cyclin A, the specific groove in cyclin $C$ is not hydrophobic, but rather negatively charged, reflecting the difference in the substrate of the corresponding kinases. The specific patch in cyclin C could bind the CTD, to increase its local concentration at the kinase. Alternatively, the patch could bind additional subunits of the Mediator CDK8-cyclin C module, MED12 or MED13, and thus be important for formation of this Mediator module.

\section{Possible mechanisms of CDK8 activation}

CDKs are generally activated in two steps: binding of the cyclin, and phosphorylation of a threonine residue in the CDK activation segment (T160 in human CDK2). ${ }^{35}$ Interaction of the phosphothreonine with three conserved arginine residues (Figure 4(b)) triggers a conformational change that results in full kinase activation. In all CDKs, the phosphorylation site consists of a threonine or serine residue, but CDK8 carries aspartate at this position (D176 in S. pombe). It is uncertain whether this aspartate residue mimics a phosphothreonine residue and interacts with the arginine residues of CDK8 (Figure $4(\mathrm{c})$ ), because the CDK8 activation segment is three residues longer than in CDK2 and should fold differently. Indeed, the activation segment of CDK7 adopts a
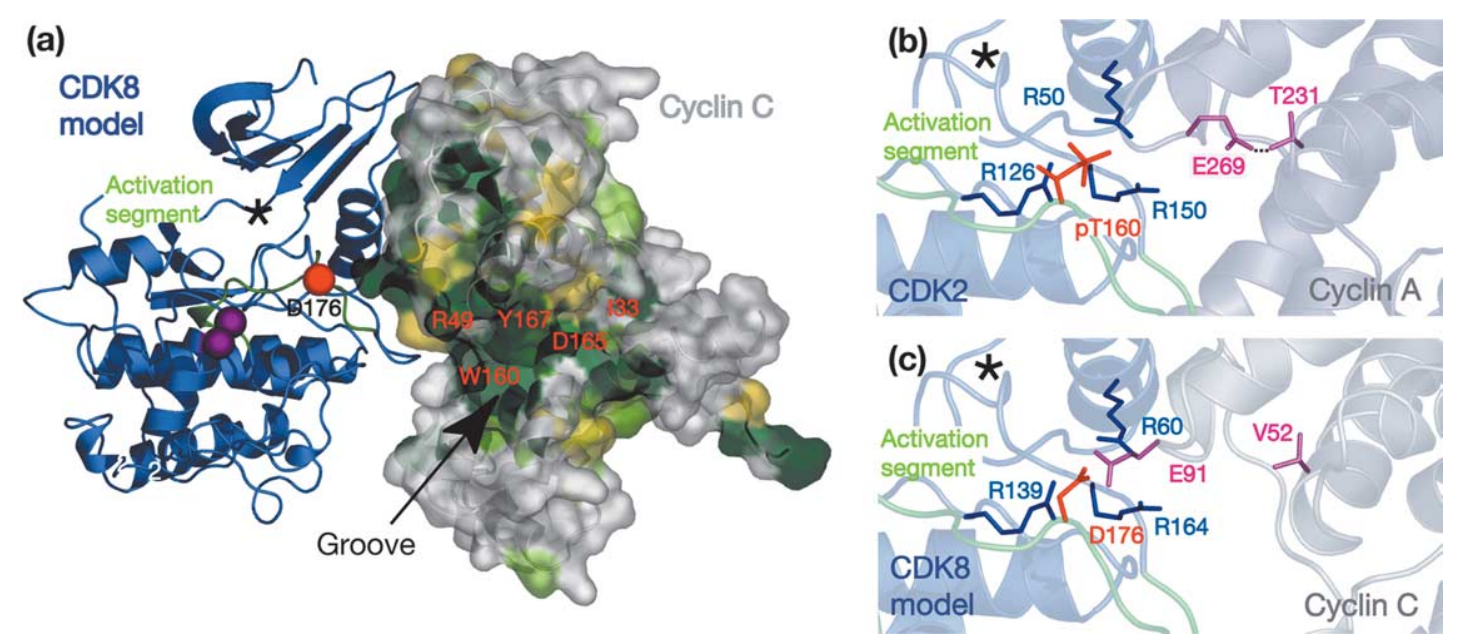

Figure 4. Structural features relating to CDK8 activity. (a) Model of the CDK8-cyclin C complex in Figure 3(a). The model is shown as a ribbon diagram, with a semitransparent surface coloured according to conservation of cyclin $C$, as in Figure 1(d). The asterisk indicates the location of the kinase active site. The CDK activation segment is in green and includes the conserved aspartate D176 (red sphere). The beginning and the end point of a nine-residue CDK8-specific insertion are marked with purple spheres. The conserved surface groove in cyclin $C$ is indicated. (b) Detailed view of the region around the activation segment in the structure of cyclin A bound to CDK2 phosphorylated at residue $\mathrm{T} 160^{49}$ (PDB-code 1JST). The phosphothreonine is in red, and the three conserved arginine residues (arginine cluster) that it coordinates are in blue. A hydrogen bond between cyclin residues E269 and T231 is shown as a broken line. (c) Possible mimicry of phosphorylation in the CDK8 activation segment. The CDK8-cyclin C model in (a) is oriented as in (b). A conserved aspartate in the CDK8 activation segment that could mimic a phosphothreonine is in red (D176). A glutamate residue in cyclin $C$ that could bind the CDK8 arginine cluster in trans is in pink (E91). The hydrogen bond depicted in (b) cannot be formed, since the donor T231 in cyclin A is replaced by valine in cyclin C (V52, pink). 
conformation that is different from that in CDK2 or other CDKs. ${ }^{27}$ On the basis of the CDK8-cyclin C model, we suggest that CDK8 activation may be achieved in trans, by interaction of the side-chain of the exposed glutamate E91 in cyclin C with the arginine residues in CDK8 (Figure 4(c)). The corresponding residue in cyclin A, E269, points in the opposite direction, and forms a hydrogen bond with T231 (Figure 4(b)), which is replaced by V52 in cyclin $\mathrm{C}$ that cannot form such a hydrogen bond (Figure 4(c)). Cyclin C could thus act like a viral "supercyclin" that activates CDK6 without phosphorylation. ${ }^{36}$ The cyclin C residue E91 could alternatively help determine CDK8 substrate specificity, similar to a conserved glutamate in an activator protein of CDK5. ${ }^{37}$

\section{Materials and Methods}

\section{Protein preparation}

The gene for cyclin C was amplified from S. pombe cDNA by PCR, and was cloned into vector pET28d (Novagen). Variants of cyclin $\mathrm{C}$ were expressed for 15 hours at $18^{\circ} \mathrm{C}$ in Escherichia coli BL21 (DE3) RIL (Stratagene). Cells were harvested by centrifugation, resuspended in buffer A (50 mM Tris (pH 8.0), $300 \mathrm{mM}$ $\mathrm{NaCl}, 10 \%$ (v/v) glycerol, $10 \mathrm{mM} \beta$-mercaptoethanol $1 \mathrm{mM}$ Pefabloc (fromRoth)), and were lysed with a French press. After centrifugation, the supernatant was loaded onto a Ni-NTA column (Qiagen) equilibrated with buffer A. After washing with $30 \mathrm{ml}$ of buffer $\mathrm{A}$, bound protein was eluted with buffer A containing $300 \mathrm{mM}$ imidazole. The protein was purified further by anion-exchange chromatography (MonoQ, Amersham). The column was equilibrated with buffer B (20 mM Tris ( $\mathrm{pH} 8.0), 100 \mathrm{mM}$ $\mathrm{NaCl}, 10 \%$ (v/v) glycerol, $3 \mathrm{mM} \mathrm{DTT}$ ), and the protein was eluted with a gradient of ten column volumes from $100 \mathrm{mM}$ to $500 \mathrm{mM} \mathrm{NaCl}$. After concentration, the sample was subjected to gel-filtration (Superose-12 HR, Amersham) in buffer B. Peak fractions were pooled and concentrated to $5 \mathrm{mg} / \mathrm{ml}$.

\section{Crystallization and limited proteolysis}

Crystals of full-length cyclin $\mathrm{C}$ were grown at $20^{\circ} \mathrm{C}$ with the hanging-drop method, using as reservoir solution $250 \mathrm{mM}$ magnesium formate, $25 \%$ (v/v) glycerol, $10 \mathrm{mM}$ DTT. The crystals reached a maximum size of $70 \mu \mathrm{m} \times 70 \mu \mathrm{m} \times 70 \mu \mathrm{m}$, and could be flash-cooled directly from the hanging drop. Deletion of the C-terminal hexahistidine tag resulted in an insoluble protein. We carried out limited proteolysis to detect flexible regions that may be removed. Treatment of purified full-length cyclin $C$ with subtilisin, followed by Edman sequencing of the obtained protein bands, revealed that the $\mathrm{N}$-terminal region of cyclin $\mathrm{C}$ is proteolytically sensitive, and is cut after residues 4 and 29. Variants of cyclin $C$ that lacked four or 29 residues from the $\mathrm{N}$ terminus of cyclin $\mathrm{C}$ were subcloned into $\mathrm{pET} 28 \mathrm{~d}$ vector. The variant comprising residues 5-228 was highly soluble and could be crystallized using a reservoir solution containing $1.25 \mathrm{M}$ sodium acetate $(\mathrm{pH} 6.5), 100 \mathrm{mM}$ imidazole, $7.5 \%(\mathrm{w} / \mathrm{v})$ PEG 400, $10 \mathrm{mM}$ DTT. These crystals grew larger and more reproducibly. Crystals exhibited a trigonal bipyr- amidal shape and grew to a maximum diameter of $150 \mu \mathrm{m}$. Crystals were harvested in the reservoir solution. The PEG 400 concentration was increased to $25 \%$ in six steps using an incubation time of one hour after each step. Crystals were incubated overnight at $4{ }^{\circ} \mathrm{C}$, and were plunged into liquid nitrogen for storage.

\section{Structure determination and refinement}

The cyclin C variant 5-228 double mutant L60M/ L186M, which contains methionine at the positions of two conserved leucine residues, was constructed with the PCR overlap extension method. Selenomethionine was incorporated as described, ${ }^{38,39}$ and a selenomethioninelabelled crystal was used in a MAD experiment at the Swiss Light Source (Table 1). Data were processed with DENZO and SCALEPACK, ${ }^{40}$ except the native dataset, which was processed with MOSFLM and SCALA. ${ }^{41}$ Program SOLVE detected four selenium sites (corresponding to residues M60, M64, M174, and M186), and was used for MAD phasing (Z-score 20.3, figure of merit 0.58). ${ }^{42}$ Phases were improved with SHARP. ${ }^{43}$ Despite the limited resolution of $3.5 \AA$, the resulting electron density map was of good quality and allowed for positioning of a polyalanine model of the cores of the two helical cyclin repeats. Subsequent phase combination and phase extension to $3.0 \AA$ with amplitudes from native data allowed building of an atomic model. Refinement of the structure with CNS resulted in a free $R$-factor of $28.8 \%$ (Table 1). ${ }^{44}$ In the refined structure, $98.2 \%$ of the residues fall in allowed regions of the Ramachandran plot, and none of the residues is found in a disallowed region. ${ }^{45}$

\section{Protein Data Bank accession code}

The atomic coordinates and structure factor amplitudes of $S$. pombe cyclin $C$ have been deposited with the RCSB Protein Data Bank with accession code 1ZP2.

\section{Acknowledgements}

This work was supported by the Deutsche Forschungsgemeinschaft, the Fonds der Chemischen Industrie, and the EMBO Young Investigator Programme. Part of this work was performed at the Swiss Light Source (SLS), Paul Scherrer Institut, Villigen, Switzerland. We thank C. Schulze-Briese and co-workers for help at SLS. We thank Anton Meinhart for help.

\section{References}

1. Dynlacht, B. D. (1997). Regulation of transcription by proteins that control the cell cycle. Nature, 389, 149-152.

2. Murray, A. W. (2004). Recycling the cell cycle: cyclins revisited. Cell, 116, 221-234.

3. Bregman, D. B., Pestell, R. G. \& Kidd, V. J. (2000). Cell cycle regulation and RNA polymerase II. Front. Biosci. 5, D244-D257.

4. Coin, F. \& Egly, J. M. (1998). Ten years of TFIIH. Cold Spring Harbor. Symp. Quant. Biol. 63, 105-110. 
5. Price, D. (2000). P-TEFb, a cyclin-dependent kinase controlling elongation by RNA polymerase II. Mol. Cell. Biol. 20, 2629-2634.

6. Liao, S. M., Zhang, J., Jeffery, D. A., Koleske, A. J., Thompson, C. M., Chao, D. M. et al. (1995). A kinasecyclin pair in the RNA polymerase II holoenzyme. Nature, 374, 193-196.

7. Tassan, J. P., Jaquenoud, M., Leopold, P., Schultz, S. J. \& Nigg, E. A. (1995). Identification of human cyclindependent kinase 8 , a putative protein kinase partner for cyclin C. Proc. Natl Acad. Sci. USA, 92, 8871-8875.

8. Borggrefe, T., Davis, R., Erdjument-Bromage, H., Tempst, P. \& Kornberg, R. D. (2002). A complex of the Srb8, -9, -10, and -11 transcriptional regulatory proteins from yeast. J. Biol. Chem. 277, 44202-44207.

9. Samuelsen, C. O., Baraznenok, V., Khorosjutina, O., Spahr, H., Kieselbach, T., Holmberg, S. \& Gustafsson, C. M. (2003). TRAP230/ARC240 and TRAP240/ ARC250 mediator subunits are functionally conserved through evolution. Proc. Natl Acad. Sci. USA, 100, 6422-6427.

10. Boube, M., Joulia, L., Cribbs, D. L. \& Bourbon, H. M. (2002). Evidence for a mediator of RNA polymerase II transcriptional regulation conserved from yeast to man. Cell, 110, 143-151.

11. Liu, Y., Ranish, J. A., Aebersold, R. \& Hahn, S. (2001). Yeast nuclear extract contains two major forms of RNA polymerase II mediator complexes. J. Biol. Chem. 276, 7169-7175.

12. Chang, Y. W., Howard, S. C. \& Herman, P. K. (2004). The Ras/PKA signaling pathway directly targets the Srb9 protein, a component of the general RNA polymerase II transcription apparatus. Mol. Cell. 15, 107-116.

13. Fryer, C. J., White, J. B. \& Jones, K. A. (2004). Mastermind recruits $\mathrm{CycC}: \mathrm{CDK} 8$ to phosphorylate the Notch ICD and coordinate activation with turnover. Mol. Cell. 16, 509-520.

14. Hengartner, C. J., Myer, V. E., Liao, S.-M., Wilson, C. J., Koh, S. s. \& Young, R. A. (1998). Temporal regulation of RNA polymerase II by Srb10 and Kin28 cyclindependent kinases. Mol. Cell. 2, 43-53.

15. Akoulitchev, S., Chuikov, S. \& Reinberg, D. (2000). TFIIH is negatively regulated by cdk8-containing mediator complexes. Nature, 407, 102-106.

16. Chi, Y., Huddleston, M. J., Zhang, X., Young, R. A., Annan, R. S., Carr, S. A. \& Deshaies, R. J. (2001). Negative regulation of Gcn4 and Msn2 transcription factors by Srb10 cyclin-dependent kinase. Genes Dev. 15, 1078-1092.

17. Nelson, C., Goto, S., Lund, K., Hung, W. \& Sadowski, I. (2003). Srb10/Cdk8 regulates yeast filamentous growth by phosphorylating the transcription factor Ste12. Nature, 421, 187-190.

18. Vincent, O., Kuchin, S., Hong, S. P., Townley, R., Vyas, V. K. \& Carlson, M. (2001). Interaction of the Srb10 kinase with Sip4, a transcriptional activator of gluconeogenic genes in Saccharomyces cerevisiae. Mol. Cell. Biol. 21, 5790-5796.

19. Ansari, A. Z., Koh, S. S., Zaman, Z., Bongards, C., Lehming, N., Young, R. A. \& Ptashne, M. (2002). Transcriptional activating regions target a cyclindependent kinase. Proc. Natl Acad. Sci. USA, 99, 14706-14709.

20. Liu, Y., Kung, C., Fishburn, J., Ansari, A. Z., Shokat, K. M. \& Hahn, S. (2004). Two cyclin-dependent kinases promote RNA polymerase II transcription and formation of the scaffold complex. Mol. Cell. Biol. 24, 1721-1735.
21. Hallberg, M., Polozkov, G. V., Hu, G. Z., Beve, J., Gustafsson, C. M., Ronne, H. \& Bjorklund, S. (2004). Site-specific Srb10-dependent phosphorylation of the yeast mediator subunit Med2 regulates gene expression from the 2-microm plasmid. Proc. Natl Acad. Sci. USA, 101, 3370-3375.

22. Leopold, P. \& O'Farrell, P. H. (1991). An evolutionarily conserved cyclin homolog from Drosophila rescues yeast deficient in G1 cyclins. Cell, 66, 1207-1216.

23. Lew, D. J., Dulic, V. \& Reed, S. I. (1991). Isolation of three novel human cyclins by rescue of $\mathrm{G} 1$ cyclin $(\mathrm{Cln})$ function in yeast. Cell, 66, 1197-1206.

24. Ren, S. \& Rollins, B. J. (2004). Cyclin C/cdk3 promotes Rb-dependent G0 exit. Cell, 117, 239-251.

25. Sage, J. (2004). Cyclin $C$ makes an entry into the cell cycle. Dev. Cell. 6, 607-608.

26. Andersen, G., Busso, D., Poterszman, A., Hwang, J. R., Wurtz, J.-M., Ripp, R. et al. (1997). The structure of cyclin $\mathrm{H}$ : common mode of kinase activation and specific features. EMBO J. 3, 958-967.

27. Lolli, G., Lowe, E. D., Brown, N. R. \& Johnson, L. N. (2004). The crystal structure of human CDK7 and its protein recognition properties. Structure (Camb), 12, 2067-2079.

28. Nikolov, D. B., Chen, H., Halay, E. D., Usheva, A. A., Hisatake, K., Lee, D. K. et al. (1995). Crystal structure of a TFIIB-TBP-TATA-element ternary complex. Nature, 377, 119-128.

29. Card, G. L., Knowles, P., Laman, H., Jones, N. \& McDonald, N. Q. (2000). Crystal structure of a gamma-herpesvirus cyclin-cdk complex. EMBO J. 19, 2877-2888.

30. Jeffrey, P. D., Russo, A. A., Polyak, K., Gibbs, E., Hurwitz, J., Massague, J. \& Pavletich, N. P. (1995). Mechanism of CDK activation revealed by the structure of a cyclinA-CDK2 complex. Nature, 376, 313-320.

31. Jones, T. A., Zou, J. Y., Cowan, S. W. \& Kjeldgaard, M. (1991). Improved methods for building protein models in electron density maps and the location of errors in these models. Acta Crystallog. sect. A, 47, $110-119$.

32. Honda, R., Lowe, E. D., Dubinina, E., Skamnaki, V., Cook, A., Brown, N. R. \& Johnson, L. N. (2005). The structure of cyclin E1/CDK2: implications for CDK2 activation and CDK2-independent roles. EMBO J. 24, 452-463.

33. Schulman, B. A., Lindstrom, D. L. \& Harlow, E. (1998). Substrate recruitment to cyclin-dependent kinase 2 by a multipurpose docking site on cyclin A. Proc. Natl Acad. Sci. USA, 95, 10453-10458.

34. Loog, M. \& Morgan, D. O. (2005). Cyclin specificity in the phosphorylation of cyclin-dependent kinase substrates. Nature, 434, 104-108.

35. Pavletich, N. P. (1999). Mechanisms of cyclin-dependent kinase regulation: structures of Cdks, their cyclin activators, and Cip and INK4 inhibitors. J. Mol. Biol. $287,821-828$.

36. Schulze-Gahmen, U. \& Kim, S. H. (2002). Structural basis for CDK6 activation by a virus-encoded cyclin. Nature Struct. Biol. 9, 177-181.

37. Tarricone, C., Dhavan, R., Peng, J., Areces, L. B., Tsai, L. H. \& Musacchio, A. (2001). Structure and regulation of the CDK5-p25(nck5a) complex. Mol. Cell. 8, 657-669.

38. Budisa, N., Steipe, B., Demange, P., Eckerskorn, C., Kellermann, J. \& Huber, R. (1995). High-level biosynthetic substitution of methionine in proteins 
by its analogs 2-aminohexanoic acid, selenomethionine, telluromethionine and ethionine in Escherichia coli. Eur. J. Biochem. 230, 788-796.

39. Meinhart, A., Blobel, J. \& Cramer, P. (2003). An extended winged helix domain in general transcription factor E/IIEalpha. J. Biol. Chem. 278, 48267-48274.

40. Otwinowski, Z. \& Minor, W. (1996). Processing of $X$-ray diffraction data collected in oscillation mode. Methods Enzymol. 276, 307-326.

41. Collaborative Computing Project Number 4. (1994). The CCP4 suite: programs for protein crystallography. Acta Crystallog. sect. D, 50, 760-763.

42. Terwilliger, T. C. (2002). Automated structure solution, density modification and model building. Acta Crystallog. sect. D, 58, 1937-1940.

43. La Fortelle, E. d. \& Bricogne, G. (1997). Maximumlikelihood heavy atom parameter refinement for multiple isomorphous replacement and multiwavelength anomalous diffraction methods. Methods Enzymol. 276, 472-494.

44. Brunger, A. T., Adams, P. D., Clore, G. M., DeLano, W. L., Gros, P., Grosse-Kunstleve, R. W. et al. (1998).
Crystallography \& NMR system: a new software suite for macromolecular structure determination. Acta Crystallog. sect. D, 54, 905-921.

45. Laskowski, R. A., MacArthur, M. W., Moss, D. S. \& Thornton, J. M. (1993). PROCHECK: a program to check the stereochemical quality of protein structures. J. Appl. Crystallog. 26, 283-291.

46. Thompson, J. D., Higgins, D. G. \& Gibson, T. J. (1994). CLUSTAL W: improving the sensibility of progressive multiple sequence alignment through sequence weighing, positions-specific gap penalties and weight matrix choice. Nucl. Acids Res. 22, 4673-4680.

47. Kabsch, W. \& Sander, C. (1983). Dictionary of protein secondary structure: pattern recognition of hydrogenbonded and geometrical features. Biopolymers, 22, 2577-2637.

48. Barton, G. J. (1993). ALSCRIPT: a tool to format multiple sequence alignments. Protein Eng. 6, 37-40.

49. Russo, A. A., Jeffrey, P. D. \& Pavletich, N. P. (1996). Structural basis of cyclin-dependent kinase activation by phosphorylation. Nature Struct. Biol. 3, 696-700.

Edited by R. Huber

(Received 8 April 2005; received in revised form 17 May 2005; accepted 19 May 2005) 